\title{
La informática educativa: Presente y Futuro
}

\author{
José Luis Rodríguez
}

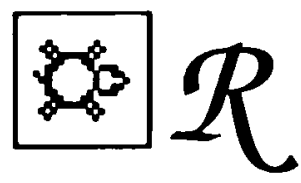

En este artículo se bace una revisión, para el lector de nuestro pais, del papel que ba jugado y puede jugar el ordenador en educación, que aun con validez general, sigue también muy de cerca las tendencias y problemas caracteristicos de lo que ba ocurrido en España. Pero se plantea también los grandes problemas de futuro: ¿qué recursos ofrece o permite boy el ordenador?, ¿cómo utilizarlo conociendo sus mejores posibilidades del mañana?

\section{REVISION DEL AREA: LA INFORMATICA EDUCATIVA EN EL UMBRAL DE LOS AÑOS 90}

Como con otras tecnologías, aunque quizás de manera más acusada, la informática ha sido y es el espejismo de un sistema de escolarización (el español, pero en general el occidental) cada vez más abrumado por sus contradicciones internas e históricas. Si hace poco fue la imagen, ahora es el bit: $-\mathrm{y}$ dentro de nada, ya mismo, será la unión de ambos-. No es de extrañar la incredulidad de muchos enseñantes frente a este mundo de promesas redentoras: preferirían que sus alumnos supieran hablar y escribir con precisión, antes que manejar vídeos y microordenadores.

Y, por otra parte, la fuerza misma de los hechos, de la total e irreversible imposición de las máquinas en estos días, hace que la misma discusión de unos años resulte hoy inútil y completamente trasnochada. Pues, efectivamente, el Basic no es ya el futuro, aquello para lo que hay que prepararse, sino que pertenece al presente más actual, casi al presente ya un poco desfasado... Pasa ahora lo que pasó con la televisión y las discusiones de los años sesenta: el propio entorno social se ha transformado, haciéndose más televisivo, siendo construido por la propia televisión.

La cuestión formal de la necesidad de enseñar informática, a usar los ordenadores, parece hoy ya muy asumida, quizás no plenamente discutida pero desde luego cada vez menos negada, por el colectivo de enseñantes. Esta 
discusión/asunción ha ido emparejada, en los últimos cinco años aproximadamente, de un fenómeno muy representativo de la introducción de la informática en el sistema educativo y que queremos comentar por su complejidad, pues ha supuesto el cambio de actitud de los enseñantes en relación a un tema que todavía puede ser discutido. Nos referimos más que a un fenómeno concreto a un conjunto de circunstancias que han cambiado y que han hecho cambiar la posición de muchos enseñantes en relación a la informática y sobre todo a su inevitabilidad escolar, circunstancias que quisiéramos comentar, o señalar al menos las que parecen más relevantes.

\section{El relativo fracaso de la EAO}

Frente al panorama de los viejos años sesenta, con mainframes incluidos, y la totalidad de los setenta - con el nacimiento de los microordenadores-, los últimos años han supuesto un acusado descenso de este viejo ideal: utilizar los ordenadores para enseñar conocimientos básicos, repetitivos, destrezas, razonamientos simples, etc. En nuestro país, el abandono (verbal y en muchas ocasiones real) de los supuestos más conductistas de determinadas pedagogías tradicionales ha ido en paralelo a este abandono del espejismo de la EAO. A este abandono también ha contribuido como causa principal la falta de programas de calidad, en parte por falta de un mercado lucrativo para los programadores, habiéndose concentrado el mercado en los juegos (para máquinas pequeñas) y en las aplicaciones para negocios. También ha contribuido la implantación en las escuelas de un estándard de ordenador que no contaba con software didáctico.

\section{La aparición de un estándard}

Sólo desde 1981, con la aparición del IBM PC y la adopción del sistema operativo de Microsoft (Ms-DOS), se puede hablar de un estándar en el terreno de los microordenadores. La famosa y malentendida compatibilidad. De hecho, todos los ordenadores introducidos en escuelas públicas españolas utilizan básicamente ese sistema operativo y son «compatibles» (Olivetti, Bull, IBM, etc.).

Sin embargo, debe hacerse una salvedad importante: esto no ha sido así en los dos países más adelantados (descontando a Japón) en el terreno informático: en Estados Unidos, durante finales de los setenta y toda la década de los ochenta, las escuelas han utilizado un ordenador más pequeño y menos potente, pero con miles de programas hechos y un buen soft educativo: el Apple II. Nacido con menos de 48k de memoria RAM, posteriormente con 64 y luego con 128, sólo recientemente se ha convertido en un ordenador de 16 bits con muy altas capacidades gráficas y de sonido (Apple HGS). Una mala política de importación y difusión de esta máquina, unida a unos precios excesivos, sin aportar la castellanización del software existente, ha hecho que evidentemente sea una máquina olvidada en el terreno escolar. En segundo lugar, el caso británico que es un modelo completamente aparte: inclusive el ordenador, especialmente construido y casi no comercializado fuera de ese país, el BBC (incluso menos potente que el Apple II). El triunfo del BBC en las escuelas inglesas se debe, de nuevo, al software especialmente creado - para un ordenador que, por poner un ejemplo, originalmente no soportaba más de 40 columnas en modo texto, pero al que se podían añadir chips y «tarjetas» que contenían el paso a 80 columnas más un procesador de textos integrado. 
Pero, en cualquier caso, es evidente que la implantación de los entornos MsDOS como estándar escolar ha supuesto varias cosas:

1) utilización de unos ordenadores con grandes aplicaciones para la gestión escolar, posibilidad de intercambio de datos entre escuelas, así como disposición de una amplia gama de lenguajes de programación y software de propósito general. Ordenadores modulares, que han permitido su ampliación básica, así como la utilización de placas y periféricos especialmente diseñados.

2) ausencia de software didáctico y castellanizado - como ya hemos apuntado.

3) utilizar un estándar muy potente en su momento (1981) pero que ha evolucionado menos de lo esperado. La evolución ha sido escasa tanto en lo referente a las máquinas (que normalmente tienen limitaciones muy serias en cuanto a la memoria RAM, la velocidad del procesador, la incorporación de gráficos, etc.), como en lo referente al propio sistema operativo, apenas modificado en sus distintas versiones, especialmente en lo que afecta al interfaz de usuario.

Quizás podríamos resumir estas consecuencias en apenas una muy simple: la utilización de una máquina pensada para la gestión empresarial, dotada de un procesador y de un software muy orientados al tratamiento de datos numéricos, que ha sido implantada en la escuela. Esto ha tenido grandes y buenas consecuencias en su momento, aunque también ha arrastrado una fuerte inversión económica en un tipo de máquina no pensada para el mercado educativo.

\section{La aparición del lenguaje LOGO}

Junto al abandono del conductismo, el «piagetismo» ha hecho su entrada en el mundo informático de la mano de Papert y el Logo. Este lenguaje ha hecho mucho por renovar esperanzas entre los maestros, aunque por ello mismo ha contribuido a renovar y crear nuevos desánimos. No sólo no está demostrada su potencial capacidad logogenésica, sino que tampoco el fomento de capacidades cognitivas más elementales puede contarse entre sus méritos incuestionados. Más aún: su inclusión curricular y sobre todo su didáctica son todavía cuestión abierta: parece ser que los niños y su pulsión/pasión epistemofilica tienen unos límites mayores de lo esperado, a la vez que más concretos y más icónicos - sin que ello quiera decir que las listas les repelan.

Por otra parte, la problemática de los efectos cognitivos de los lenguajes de programación sobre los sujetos debe matizarse: sólo si tales efectos están muy acotados y medidos la investigación puede tener sentido. Decir que el Logo (o cualquier otro lenguaje) «ayuda a pensar», o frases más o menos parecidas, es como decirlo del latín, de las matemáticas, de la literatura y el lenguaje, o incluso de las horas dedicadas a dibujo. Todo ayuda a pensar, y probablemente ninguna actividad ayuda más que otra a pensar «en general». Si lo que Papert y otros defensores del Logo pretenden decir es que ayuda al desarrollo de, por ejemplo, la capacidad de razonamiento inductivo, eso es algo muy anterior. $\mathrm{Ha}$ blar en términos generales puede conducir a una sobrevaloración excesiva de actividades que parece contraria a cualquier otra que piense el proceso educativo más como el resultado de procesos sinérgicos que como la resultante de un único proceso principal (por importante que sea).

Al menos estos tres factores, más entrelazados de lo que parece, habrían determinado la asunción por parte de los enseñantes de la inevitabilidad informática: 
1) poco (y malo) software didáctico, lo que hacía desaparecer la angustia del ordenador como sustituto del maestro.

2) la implantación real de un estándar escolar en la vorágine del mercado informático.

3) la aparición de una aplicación informática congruente con las ideologías pedagógicas del momento.

El desaliento de muchos enseñantes también aparece, al menos en parte, por razones derivadas de estos mismos tres factores:

\section{El fracaso de la $E A O$}

Al no haber software didáctico (bueno y mucho), la utilización educativa de los ordenadores ha tenido que replantearse - iafortunadamente! - Por desgracia, lo primero que se intentó fue, quizás porque el sistema educativo actuó movido por la fascinación de las nuevas y bien retribuidas profesiones. Enseñar a programar: Basic y Pascal. El cambio, lentamente, apuntó hacia el uso de las nuevas tecnologías: que los escolares supieran utilizar un tratamiento de textos o una base de datos (para lo que los ordenadores tipo IBM-PC han dado un muy buen juego), así como otras posibilidades más complejas y también muy interesantes: hojas de cálculo, telecomunicaciones, control de aparatos en un laboratorio, etc.

Quizá hoy día empiece a apreciarse tanto la importancia y necesidad de este cambio en la concepción educativa de la informática como su insuficiencia o desaprovechamiento en las posicilidades didácticas de los ordenadores. Por una parte, desimplicó la enseñanza de la informática de la obligatoriedad de aprender lenguajes de programación (incluso pensados como fin en sí mismos), relativizando la conexión entre aprendizaje de la programación y mayor desarrollo de capacidades intelectuales; por otra, enfatizó lo que siempre debía haber resultado obvio: que lo que hace que cualquier material pueda considerarse «educativo", es el tratamiento pedagógico que de él se hace, y que, por tanto, las aplicaciones de propósito general eran perfectamente válidas como objeto y objetivo de aprendizaje; y finalmente replanteó el tema del software concebido como didáctico, esto es: creado especialmente con intenciones didácticas, al dejarlo reducido a un segundo plano (dada su escasez y no muy alta calidad).

Conviene detenerse en estas consecuencias (¿o causas?), en tanto en cuanto están mutuamente implicadas y en tanto en cuanto deben matizarse más. Pues es cierto que la introducción de los lenguajes de programación en la escuela responde a un espejismo social (una nueva profesión, bien pagada en el mercado, con escasez de profesionales, de gran prestigio intelectual, y con el diseño futurista de un paisaje repleto de máquinas controladas por hábiles programadores), pero también es cierto que ello mismo es independiente tanto de: (a) las posibles virtudes logogenésicas del aprendizaje de un lenguaje de programación, como de (b) el modelo pedagógico de la enseñanza de la programación (tanto de carácter general: lo que supone decantarse por lenguajes en la selección/diseño curricular, como de los modelos didácticos aplicados). Dicho de otro modo: es muy posible que el aprendizaje de lenguajes de programación reporte parecidos beneficios a los del aprendizaje de otro tipo de materias (por ej., lógica, matemáticas) que son supuestamente estructurantes de capacidades cognitivas ligadas a la abstracción y al razonamiento complejo, y ello independientemente de 
la mala introducción de tales lenguajes en el ámbito curricular. Es algo así como si se hubiera presentado la necesidad de estudiar matemáticas de un determinado nivel elevado como condición de una profesión bien remunerada al acabar la escolarización. Sin embargo, conviene dar otra vuelta de tuerca más: no hay necesidad alguna de incluir los lenguajes de programación como forma de entrada de la informática en el currículum escolar. Casi al contrario, parece mucho más sensato postular que la enseñanza de la informática pasa por convertir a los alumnos en usuarios (Rodríguez Illera, 1986).

En resumen, la inexistencia de EAO de calidad ha hecho que la escuela olvidase la función primaria de la ya lejana introducción de los ordenadores: la de enseñar con y a través suyo, para dedicarse a un replanteamiento necesario y pedagógicamente más interesante. En realidad se ha tratado de un diversificación, hoy ya inevitable, que ha venido de la mano de la existencia y desarrollo de la microinformática y ordenadores personales, en especial en los últimos diez años, y más específicamente en los últimos cinco (cuando las máquinas han bajado sus precios a la vez que aumentaban sus prestaciones).

Este fenómeno se ha producido en el sistema educativo, pero es obvio que se ha producido también y de manera más generalizada en el conjunto de la sociedad: los micros se venden hoy como un electrodoméstico más, en tiendas no especializadas, grandes almacenes, etc. De una situación de carencia de ordenadores se ha pasado a otra en la que se mide públicamente la eficacia administrativa (del sistema judicial, por ejemplo) en términos de la cantidad de recursos informáticos que utiliza, en la que es habitual encontrar microordenadores en una mayoría de hogares, normalmente ligados a los hijos en edad escolar, pero también a los padres que lo utilizan como un complemento de su trabajo en la oficina. Esto es, como es de esperar y como proponemos ${ }^{1}$, han sido probablemente los sectores terciarios de nuestra sociedad, los que están directamente implicados en la manipulación de la información, aquellos que han asumido la utilización de ordenadores en el ámbito familiar.

\section{El estándard de bardware y de sistema operativo}

Si los ordenadores domésticos mayoritarios siguen siendo hoy máquinas de prestaciones muy limitadas (con una media de $128 \mathrm{~K}$ de RAM, y un máximo de una unidad de disco), concebidas especialmente para juegos y algunos programas de uso general, además de los consabidos lenguajes, no caba duda que, como ya hemos señalado, el ordenador escolar es la máquina estándar en ordenadores personales (con 512 o 650 de RAM, dos unidades de disco o incluso disco duro y en ocasiones monitor en color de mucha mayor resolución). La potencia es completamente distinta, pero también las limitaciones. De hecho, si pretendemos ahora mostrar cómo, paradójicamente, la elección de una máquina más potente también ha sido un factor que ha contribuido al desánimo de los docentes, hay que insistir en las limitaciones que impone un ordenador compatible. Estas limitaciones provienen básicamente de tres factores:

a) de la escasa evolución de la máquina desde 1981. En contra de lo que pueda parecer, las sucesivas ampliaciones de los ordenadores personales (XT, $\mathrm{AT})$ no han supuesto cambio alguno en las posibilidades de la máquina - a excepción, obviamente, de la mayor velocidad--. En el mercado microinformático son demasiados años para no cambiar. Muchos docentes han visto cómo 
aparecían otras marcas de ordenadores, y, en general, cómo los microordenadores podían manejar mucho mejor la información gráfica que las máquinas destinadas a su escuela. Afortunadamente, con las placas gráficas y los aceleradores adecuados cualquier ordenador antiguo logra una potencia mucho mayor (sobre todo si se le proporciona un monitor de mayor resolución), si bien es cierto que se trata de un arreglo económicamente costoso. Los nuevos ordenadores basados en la misma familia de chips, la serie PS, añade lo que parece completamente necesario: un monitor de alta resolución tipo fósforo blanco, discos de 3,5 (que son rígidos, de mayor capacidad y más manejables), una mayor velocidad de procesamiento - a partir del modelo 50 -, y mayores capacidades gráficas. Por desgracia, se vuelve a tratar de una solución económicamente cara.

b) el verdadero punto crítico de estas máquinas ha sido sin duda el interfaz que utilizaban para comunicarse con el usuario. Esto es, el sistema operativo Ms-DOS. Cualquier acción que el usuario quiera realizar pasa necesariamente por teclear correctamente una o varias instrucciones. Del mismo modo, la pantalla del ordenador es tratada como una hoja de papel enrollado, en la que las órdenes actuales desplazan a las anteriores, y los contenidos de un determinado directorio desaparecen si son demasiado largos. En relación a esta concepción no se puede decir que los enseñantes hayan reaccionado bien; como es lógico, no es lo mismo usar el teléfono que usar un sistema operativo. Se puede decir que éste es incluso innecesariamente desagradable para el usuario. Muchas aplicaciones han recurrido a la única salida: ofrecer un sistema de comunicación usuario-máquina que explicite la jerarquía de niveles y de operaciones a través de la que se mueve el usuario mediante un sistema de menús, con opciones prefijadas desde luego, pero en los que la elección del usuario se limite a señalar o a marcar lo que quiere hacer, sin preocuparse de cómo hacerlo. Sin embargo y sorprendentemente, sólo mediante utilidades de otras casas comerciales es posible trabajar sobre el Ms-DOS desde una perspectiva de menús. Utilidades que no todos los usuarios conocen y que, desde luego, están en inglés.

El interfaz ha sido una barrera inicial muy fuerte. Incluso cuando se aprende un tratamiento de textos, sólo se aprende el tratamiento de textos y es necesario aprender el sistema operativo aparte. En general, hoy parece claro que no es la manera adecuada para comunicarse con un ordenador. Todas las innovaciones de los últimos años han arrancado precisamente de un replanteamiento del interfaz de usuario, tanto en lo que afecta a los medios de introducción (pantallas táctiles, lápices, ratón, etc.), como al propio interfaz (menús, iconos, pantallas movibles, etc.). Sólo nuevos sistemas operativos van a permitir que estos ordenadores se transformen y reconviertan (tipo GEM, Windows, etc.), si bien falta que exista un software adecuado y generalizado.

c) en cualquier caso, el tercer aspecto relativo a los ordenadores compatibles es el tipo de software que han generado. De los varios miles de programas «didácticos» que componen las librerías de MS-DOS, lo cierto es que pocos han sido usados de manera habitual en entornos educativos. Las razones son obvias: escasa utilización de gráficos, interfaz de usuario poco amigable, contraintuitivos, en algunos casos necesidad de conocer el sistema operativo mínimamente, entrada exclusivamente por teclado, flujo lineal del aprendizaje, muy dirigidos por el autor, etc. Aunque no todas estas características negativas sean compartidas por todos los programas, la mayoría de ellas sí lo son por la mayoría de ellos. Tanto las referidas a aspectos pedagógicos como las que se derivan del interfaz de estas máquinas. 
d) Finalmente, la existencia de un lenguaje congruente con la ideología pedagógica dominante (la actividad del educando, tal y como es pensada en el paradigma piagetiano), firmemente defendido y ensalzado a través de obras clásicas (Papert, Reggini, etc.), y sobre el que se han vertido auténticos ríos de tinta.

Es muy posible que, de modo parecido a como ha pasado con los otros dos factores, también la importancia concedida al Logo haya tenido un efecto de rebote al no haberse confirmado el gran número de esperanzas en él depositadas. Ello independientemente de las afirmaciones de su creador: la cuestión está en el clima que se ha creado en torno a un lenguaje. Por ejemplo, para ciertos defensores del Logo, casi la única actividad que el alumno debería realizar con el ordenador pasaría por el desarrollo de proyectos en ese entorno de programación. No es cuestión aquí el examinar tales u otras pretensiones. Al contrario, el asunto central es ver cómo el exceso de optimismo ha conllevado una dura prueba de realidad: los alumnos tienen problemas (y serios) para continuar indefinidamente explorando el micromundo de la geometría de la tortuga, el acceso al trabajo con listas es algo completamente distinto a lo anterior (sólo queda la idea de recursividad bien aprendida) y no se trata de un entorno de programación perfectamente adecuado para proyectos complejos. Dicho de otra manera, el lenguaje Logo es ante todo un lenguaje de programación, y como tal puede recibir la mayoría de las críticas dirigidas a los lenguajes: existen las aplicaciones de propósito general, el software especialmente diseñado, etc. Las distintas versiones de Logo no sólo no mejoran nada la cuestión, sino que paradójicamente pueden agravarla; por ejemplo, versiones más potentes, dirigidas a objeto, como ObjectLogo de Coral Software, son más complejas; o el Logo en tres dimensiones también es mucho más complejo.

El problema es sencillo: detrás del razonamiento pedagógico con el que el Logo ha entrado en el sistema educativo está la cuestión obvia de que se trata de un lenguaje de programación. Los enseñantes lo han comprendido y lo han vivido: después de aprender a hacer un cuadrado, o una espiral, o un proyecto algo más complejo, después de lograr que sus alumnos realicen proyectos y que dominen la parte geométrica del lenguaje, son los propios profesores los que se encuentran ante el problema de utilizar un lenguaje que es complejo, que no está diseñado para la programación en general, que si se pretende hacer más potente es a costa de hacerlo más complejo, y que tarde o temprano acaban por abandonar (de la misma manera que abandonan el Pascal o cualquier otro lenguaje). No a todo el mundo le gusta programar -o no todas las personas tienen las capacidades cognitivas adecuadas, o bien el aprendizaje les resulta demasiado costoso.

Es obvio que el desengaño proviene de otros muchos factores (por ej. la falta de tiempo real para aprender a utilizar las máquinas, la no contemplación en el currículum de la informática educativa, la no contabilización de las horas dedicadas, la falta de apoyo en núcleos de población pequeños, la dificultad encontrada para programar, etc.), además de los comentados y relativos a las limitaciones de las máquinas elegidas, la ausencia de buena $E A O$ y las dificultades del Logo para ir mucho más allá de las fases iniciales.

Nunca como ahora las administraciones públicas habían invertido tantos recursos de forma tan planificada en tema alguno relacionado con la tecnología (piénsese en la casi inexistencia de planes referentes a los audiovisuales, o los escasos recursos de que disponen cuando existen). No es lugar para evaluar la forma como se han invertido tantos recursos, el propio diseño pedagógico (cuando 
lo ha habido) que ha guiado los mencionados planes, o la evaluación que se ha hecho del impacto que los ordenadores han tenido en los centros. Es suficiente constatar que parte del optimismo y del convencimiento sobre la inevitabilidad informática provienen muy directamente de la inversión realizada por los diferentes gobiernos autónomos o por el propio MEC. Y también es suficiente haber intentado mostrar cómo existe un cierto desencanto generado en gran parte por la poca sensibilidad pedagógica hacia la cuestión (a veces parece como si todos los planes sólo fuesen gigantescos distribuidores de máquinas) y en parte también por un cierto efecto «de rebote» que determinadas máquinas-sof tware y determinadas ideologías hiper-optimistas han generado. Afortunadamente, la incorporación de las tecnologías informáticas al sistema educativo es un largo proceso, que sólo está en sus inicios, y cuyas contradicciones actuales serán sólo objeto de estudio para historiadores en el próximo futuro; pero mientras todo este proceso se realiza y mientras la incorporación informática deja de ser noticia para ser uso cotidiano, todavía es necesario elaborar una polémica que implique al mayor número posible de profesores y que sitúe la problemática de la informática educativa más del lado futuro, de lo que está a punto de llegar, de cómo queremos en definitiva que la informática forme parte del sistema educativo, que del pasado más reciente por interesante que haya sido.

\section{LAS NUEVAS POSIBILIDADES Y LOS NUEVOS CONCEPTOS}

\section{El retorno de la EAO. Valoración técnica y pedagógica}

Entre las muchas novedades que ese están produciendo una de las no menores es lo que podríamos denominar «retorno» de la EAO. Retorno por cuanto ha habido una época reciente en la que no se ha visto claro, y quizás con razón, el propio sentido de la $\mathrm{EAO}$, sin embargo, por ver cómo en todos los órdenes su imposición está realizándose lenta pero imparablemente. Vamos a examinar esta cuestión más de cerca.

La propia expresión «enseñanza asistida por ordenador», supuesta versión de la inglesa «computer aided instruction», hay que entenderla en el conjunto de expresiones generadas para dar cuenta de la peculiar utilización de los ordenadores en el interior de la enseñanza reglada. De hecho, EAO, es una expresión habitualmente ligada al uso de programas informáticos específicamente hechos para ayudar al profesor en la explicación de algún tema específico de una materia; esto es, el denominado software educativo hecho a la medida exacta de las necesidades escolares («courseware»).

Ha habido históricamente, como ya hemos señalado, una cierta polémica sobre la propia necesidad y concepción de la EAO. La resolución de tal polémica no está resuelta, si bien es cierto que pasamos en la actualidad por un momento particularmente crítico. Podríamos señalar, si nos atenemos a una división analítica inicial entre críticas pedagógicas y críticas informáticas, las siguientes:

\section{Críticas pedagógicas}

1) Responder a un modelo conductista de enseñanza. La referencia obvia es que la mayoría de la EAO se ha diseñado como si se tratase de un curso de enseñanza programada. Esta crítica conlleva el cuestionamiento del propio uso de los ordenadores en una escuela cambiante y que pretende acceder a formas 
de enseñanza y de evaluación mucho más individualizadas, abiertas, flexibles, y en las que la propia posición del alumno sea mucho más activa. Se critica en definitia la posición pasiva en la que se coloca al alumno, el papel incuestionado del ordenador como garante de la verdad del conocimiento transmitido, etc. Como es obvio se trata de una crítica al modelo global de relación educativa que implica una determinada EAO (quizá la más generalizada).

2) Falta de una interacción personalizada con el alumno. Aquí la referencia es la incapacidad de la mayoría de los programas microinformáticos para contemplar en su desarrollo la asunción de que son usados por diferentes personas que están respondiendo de manera bien diferente (más adelante volveremos sobre este punto).

3) La casi inexistencia de EAO que conlleve simulación. Esto es, constatar que la mayoría de los programas son simples transmisores de conocimientos, al estilo de libros electrónicos, pero que su capacidad para simular (también electrónicamente) en un momento determinado aquello de lo que están hablando - cuando es posible hacerlo- suele ser muy limitada o nula.

4) Otra de las críticas que podría hacerse a la $\mathrm{EAO}$, desde el punto pedagógico, es su inserción curricular. Si bien es algo generalizable a otras tecnologías en alguno de sus aspectos (deben estar incluidas en el tiempo habitual de clase, o bien deben hacerse clases especiales, etc.), la microinformática y la EAO especialmente han introducido una especifidad históricamente nueva: la posibilidad (más fantaseada que real) de sustitución del profesor. Esto es, si la EAO transmite conocimientos de manera autónoma (de forma similar a lo que puede hacer un vídeo, pero no un conjunto de diapositivas sin organizar, u otras tecnologías), incluso en el peor de los casos: cuando no existe simulación, lo cierto es que plantea, por lo menos, las nuevas formas de relación que el profesor debe adoptar en la dinámica global de la clase cuando se utilizan estos programas.

5) Finalmente, por acabar un listado que podría ser muy largo, está la cuestión de la relación que el software educativo mantiene con el profesor que decide sobre su uso. La mayoría de los programas se han realizado de manera completamente externa al lugar donde van a ser utilizados, y no permiten que $\mathrm{n} 1 \mathrm{el}$ profesor ni el alumno los modifiquen, adecuándolos a sus necesidades. Se trata de programas «cerrados», por utilizar aquí la oposición entre programas abiertos y cerrados. Aunque se trata de una oposición discutible, lo cierto es que ambos polos permiten clasificar fácil y rápidamente el software en relación a su flexibilidad potencial para ser cambiado por los profesores, así como en la posibilidad de que ellos mismo puedan crear nuevos ejercicios (King 1989)2.

\section{Críticas informáticas}

Los propios profesionales de la informática han señalado muchas veces algunos defectos técnicos importantes de la mayoría del software educativo. Se pueden mencionar entre otros:

1) falta de capacidad gráfica de los microordenadores utilizados (especialmente: Apple II y compatibles IBM, cuando no utilizan una placa gráfica). Es obvio que determinadas simulaciones deben hacer intervenir necesariamente componentes gráficos (por ej., las referidas a biología, óptica, etc.), así como en general los programas de EAO ganarían mucho con un aumento considerable de la resolucón de pantalla y del color utilizado (que a veces ha sido casi contraproducente). 
2) falta de formas de comunicación persona-máquina alternativas. La casi totalidad de la comunicación se produce a través del teclado. Lo más natural sería el reconocimiento individualizado de la voz. Pero es claro que en el ámbito educativo se trabaja con microordenadores muy sencillos y que tales formas de comunicación no pueden producirse por su elevado coste económico. Otras formas alternativas (por ej., pantallas táctiles, ratón, lápiz óptico, etc.) tampoco han solido estar disponibles. En general, la crítica se referiría a un interfaz de comunicación más humano ${ }^{3}$.

3) desde el punto de vista informático podría pensarse en la existencia de microordenadores para el ámbito educativo que poseyesen capacidades de animación. Aunque es obvio que no alcanzan la calidad de la imagen producida por la señal vídeo, también lo es que gran parte del factor motivacional ligado a la EAO y en general a la microinformática se ha descuidado mucho.

Este conjunto de críticas que hemos intencionadamente separado nos muestra la complejidad del tema. La informática educativa y la EAO más especialmente son comparadas (de manera más o menos consciente, eso da igual) con todas aquellas otras tecnologías y modos de relación que previamente ocupaban el lugar que de alguna manera éstas parecen querer usurpar. Por ejemplo, si hablamos de la EAO como de algo que responde, iy mal!, a un concepto de enseñanza programada, es porque en cierta manera la comparamos con los libros/fichas prototípicos de la misma, esto es con una forma determinada del lenguaje escrito. Si decimos que no se adapta suficientemente a cada alumno en lo que tiene de individual, es porque de alguna manera la comparamos a un tutor, a una persona. Si ahora nos fijamos en su deficiente calidad gráfica o en la casi total inexistencia de animación, es porque la estamos comparando a la televisión y al vídeo. $Y$ así sucesivamente.

En todas estas comparaciones la EAO siempre pierde. Sólo se hace imprescindible cuando se trata de algo muy especializado y normalmente implementado en sistemas muy alejados por precio y calidad de los disponibles en las escuelas. Y quizá es ésta la cuestión: si la utilización del ordenador no es absolutamente única (e inimitable), siempre es posible compararlo con otras tecnologías o con las propias personas en situación desfavorable. Las razones de tal comparación son también obvias: nunca ningún avance ha despertado tal cantidad de expectativas en el campo educativo en lo que afecta a cambios profundos en la transmisión de conocimientos. Pero hoy empieza a ser obvio que el ordenador de hace apenas un par de décadas (basado sobre todo en el procesamiento de información numérica y de cadenas verbales de forma muy primitiva) no es el instrumento polivalente adecuado en el ámbito escolar; que ni tan siquiera lo es el microordenador de los años 70 , y que incluso los actuales compatibles están lejos de esa necesaria versatilidad que requiere el ámbito educativo. El microordenador que parece ser capaz de ocupar un lugar central en las escuelas y en la propia sociedad actual es una máquina varias veces superior en precio a los actuales micros compatibles, mucho más rápido, con mucha más memoria, capacidaddes gráficas y de animación, control y síntesis musical, software más potente, medios de comunicación persona-máquina más evolucionados incluyendo la síntesis, recepción y digitalización de voz, con fácil acceso a medios ópticos de almacenamiento masivo de información, y muy abierto al control tanto de sistemas de vídeodisco como de aparatos de laboratorio. Esto es, se necesitan otros diez o veinte años de investigación y desarrollo constante y rápido del mercado, a la vez que el mantenimiento de la misma tendencia a la baja de los pre- 
cios que se ha operado a lo largo de la presente década. En resumen, el ordenador adecuado para el sector educativo es el ordenador que en los próximos años se configurará como estación de trabajo a nivel universitario. Sólo en un sistema semejante la EAO creada prodría integrar varios medios simultáneamente y la potencia de cálculo, gráfica, etc., del ordenador, lo acercaría a ser un producto mucho más cercano a la calidad que en la actualidad proporcionan otras tecnologías más toscas pero más eficaces, así como las propias capacidades humanas del profesorado.

Sin embargo, también hay que plantearse la cuestión de la EAO (entendida genéricamente) desde posiciones más pedagógicas. De hecho, es obvio que la mejora de la micros disponibles acabará por introducir en las escuelas máquinas mucho más cercanas a las necesidades del sector educativo. Pero estas necesidades pasan por otros aspectos además de la potencia y tipo de máquina. No pretendemos abrir la cuestión de la inserción curricular de la informática, por más que sea muy interesante. Nos referimos a otro tipo de necesidades más concretas y más «inmanentes» a la propia relación persona-máquina: por ejemplo al propio interfaz, problema verdaderamente central y relativamente descuidado; o también, y quizás más importante desde nuestro punto de vista, a la propia noción/diseño de software eductivo y de EAO. Trataremos únicamente este último problema por ser el más cercano a la intervención pedagógica.

\section{Las nociones de software educativo y de EAO}

Ya antes se ha insistido en cómo el relativo fracaso de la EAO ha contribuido a la reorientación actual del uso educativo de los ordenadores. Sin embargo, a lo largo de la argumentación se ha presupuesto conocer en qué consistía esta manera de utilizar el ordenador para ayudar en el proceso instructivo. Y nada más lejos de la realidad: la calificación de «educativo» que se hace sobre determinados programas es una de las mayores arbitrariedades recientes en este campo. Como hemos intentado mostrar en otro lugar (Gros y Rodríguez Illera, 1985), lo que hace que un determinado programa pueda o no recibir tal calificación es simplemente el uso que de él se hace antes que la forma en que ha sido diseñado. Por tanto, y desde esta posición, tan «educativo» es enseñar a usar un procesador de textos como utilizar un CD-ROM que contenga una enciclopedia como el último programa de EAO para enseñar la circulación de la sangre. Todo dependerá de la capacidad del profesor para ofrecer un modelo coherente y motivador de inclusión del tiempo-con-ordenador en el conjunto del tiempo escolar.

Si bien esto es efectivamente así (y que el uso escolar de los programas de propósito general es un acierto), también lo es que el ordenador es además una máquina adecuada para producir material didáctico de primera calidad. No cualquier ordenador, ni cualquier programa, ni cualquier «programador-diseñador». Más adelante volverá esta cuestión, aunque de momento es preciso seguir examinando la noción misma de EAO y de software educativo.

De hecho, ambas nociones ocultan una clasificación tan prolija como contradictoria; en el caso de la EAO: ejercitación, simulaciones, tests, programas informativos, etc. En el caso del software educativo, deberíamos añadir los programas de propósito general, los juegos, etc. No parece necesario insistir en que enumeración semejante poco aporta desde el punto de vista pedagógico. Los programas son agrupados en géneros, definidos a su vez por el uso y por el propio 
mercado informático, que en cualquier caso sólo hacen referencịa a lo que el usuario real o potencial puede obtener del programa, pero no al tipo de relación educativa que permiten (lo que por otra parte es lógico, dado que se trata de clasificaciones comerciales y no pedagógicas). Puede sorprender que las reflexiones sobre el software provinientes de psicólogos y pedagogos no hayan en general ido mucho más allá de tal enumeración de géneros comerciales, pero lo cierto es que su novedad misma hace que sólo desde hace unos años sea posible empezar a pensar este tema. Una de las manera de hacerlo es considerar la cuestión del software como inmersa en la situación comunicativa que define la enseñanza (tema ya apuntado en Gros y Rodríguez Illera, 1985).

Si nos fijamos en uno de los aspectos centrales, los contenidos que se transmiten a través el software educativo, el qué se transmite, nos vamos a encontrar con problemas muy serios para poder definirlos. De hecho, la variedad de la transmisión es tan grande que es difícil tan siquiera enumerar un catálogo. Cualquier contenido de cualquier libro de texto puede ser colocado en un programa. Pero la EAO también está formada por «conocimientos» que de alguna manera no están en los libros. Por ejemplo, una base de datos conteniendo datos económicos de los países europeos no es simplemente un enorme álbum lleno de páginas con datos: el programa permite relacionar ese amplio conjunto de datos de manera fácil, permitiendo visualizar interrelaciones que en soporte papel y sin la organización que introduce la propia base de datos sería prácticamente imposible. Por tanto hay algo más que simple conocimiento, que un conjunto de datos o referencias. Hay datos y conocimientos «planos», y datos y conocimientos relacionados.

Una posible clasificación del conocimiento que se transmite y de los otros aspectos presentes a través de la $\mathrm{EAO} /$ software educativo debería atender a cómo el conocimiento está estructurado internamente y a cómo lo está en su relación con el usuario. Por ejemplo, es obvio que lo que se transmite no tiene siempre el mismo orden de generalidad: ¿cómo valorar un software que plantea problemas? Como es sabido, muchos enseñantes consideran que resolver problemas es una de las formas básicas de cualquier aprendizaje en profundidad; resolviendo problemas no sólo se asume el conocimiento, sino que el alumno demuestra ser capaz de generalizarlo a situaciones nuevas. En muchas ocasiones, el conocimiento que se transmite en la educación consiste en la simulación de un (micro) mundo, de un sistema. Qué forma mejor para explicar el funcionamiento de algo que su propia prueba y experimentación; aunque el sistema educativo no pueda casi nunca lograr para el alumno una relación de aprendiz (practicando con las propias cosas, a la vez que siendo guiado por un maestro en el uso de las mismas), tampoco parece cuestionable que la práctica con sistemas que simulan la realidad es uno de los usos centrales de la EAO (más adelante volveremos brevemente sobre este punto). $\mathrm{Y}$ de alguna manera también simular un sistema real (por ej., el juego del ajedrez, un laboratorio de electricidad, etc.) es también transmitir información, permitir que el alumno la manipule y compruebe cómo funciona.

En fin, parece que al menos tres tipos de conocimientos son transmitidos por la EAO: (1) conocimientos básicos; (2) problemas; (3) sistemas simulados.

Hay una creciente complejidad en esta escala, y no tanto porque los primeros sean poco complejos, cuanto por la forma de la transmisión. De hecho, lo que hemos denominado conocimientos básicos pueden descomponerse a su vez (desde el punto de vista de la forma) en conocimientos referenciales y relaciona- 
dos. Los referenciales son aquellos que pueden ser siempre sustituidos por una información en otro soporte (clásicamente, el libro), a veces con mucha mayor precisión, a veces no. Ejemplos de conocimientos referenciales serían las informaciones transmitidas en el interior de cualquier EAO y que sólo hiciesen mención a conocimientos ya presentes en otros media. Por el contrario, los relacionados dependen del propio soporte informático que es el que los organiza y el que les da vida.

Debe señalarse que los conocimientos relacionados pueden ser de varios tipos según el tipo de relación que se haya establecido: a) jerárquica, b) categorial, c) hipertextual. Una relación es jerárquica cuando presenta el conocimiento ordenado en niveles, explicitando claramente a qué nivel está asignada una información determinada; la representación típica es un grafo de forma arbórea. Por el contrario se denomina categorial a una presentación de la información en forma de base de datos: la información está categorizada (por campos), de manera tal que toda información pertenece a una clase o varias; el principal carácter de este tipo de organización de la información es la posibilidad de organizar búsquedas y selecciones exhaustivas de la información. La información está organizada hipertextualmente cuando las relaciones entre partes de tal información global han sido definidas previamente en función de algún criterio explícito (por ej., el propio sentido común o/y las propias pertinencias del creador del sistema hipertextual). Las ventajas de la organización hipertextual residen en ser una forma combinada de las dos anteriores, pero superando los límites de la forma jerárquica y pudiendo saltar entre niveles - que no siempre tienen porqué estar bien definidos-, a la vez que siendo mucho más flexible que la forma categorial (para más detalles, cfr. Anexo). Es obvio que los conocimientos transmitidos van cada vez más a mezclar todos estos tipo de organización de la información.

Además de la cuestión referente a los conocimientos que se transmiten está la cuestión más específica de la manera en que son transmitidos - no tanto el cómo se transmiten que es algo muy ligado a la propia definición de los conocimientos, y a lo sumo a un eje o escala de motivación para el alumno, cuanto a la relación educativa que se crea por el hecho mismo de la transmisión-. Esta es una cuestión muy poco trabajada en la tradición de la $\mathrm{EAO}$, que sólo aparece bajo planteamientos técnicos. La relación educativa de transmisión puede descomponerse (además de la selección y organización de los contenidos) en varios ejes:

1) eje de interactividad

2) eje del ritmo del aprendizaje

3) eje de la evaluación

Sólo vamos a discutir el primer eje. Se trata de caracterizar globalmente el tipo de relación que viene pre-impuesto por el software. Al decir pre-impuesto nos referimos al hecho de que si bien todo software puede ser utilizado de múltiples maneras y en múltiples contextos, también es verdad que existe en muchísimos casos una utilización «estándard» que vienen de alguna manera casi impuesta por el propio programa. Hay programas que predeterminan casi completamente las opciones del usuario y los hay que le dan un alto grado de libertad. Este primer eje se refiere a cómo un software específico de EAO delimita la relación que un usuario puede mantener con él; esto es, si bien y casi por definición cualquier programa informático es interactivo, también es cierto que 
hay grados y maneras de interactuar y que es en parte el propio software el que los determina. Básicamente, la interacción se reparte en un eje doble: por una parte, la posición activa/pasiva que le es asignada al sujeto, y por otra la cantidad y calidad de feedback existente a lo largo de la ejecución del programa.

Cuando, por ejemplo, el tipo de conocimiento que se transmite es la simulación de un sistema (i.e., la organización dinámica del sistema mismo), es obvio que el grado de interacción debe ser necesariamente alto. Cuando, por ejemplo, se transmite conocimiento referencial en formato hipertextual la interactividad es también alta (posición activa del usuario más feedback inmediato y constante), si bien se trata de una interacción que sólo responde a estrategias de ramificación no-lineal (hipertextual) más o menos complejas. Ambos casos nos muestran dos tipos distintos de interacción, y, en cualquier caso, una interacción distinta a aquella en la que el programa analiza la respuesta/elección del usuario para actuar en consecuencia.

Dicho de otra manera, el lazo o camino que conecta un nodo o botón con otro es obviamente el fundamento de la posible interacción. Sin embargo, hay uniones simples, del tipo:

«Al elegir la opción "B2" ir a F3"

"If variable "answertime" is greater than 10 , then goto...»

y uniones mucho más complejas para las que la elección de una determinada posibilidad sólo hace que iniciar un proceso complejo de decisión, en el que pueden entrar todas las técnicas de la inteligencia artificial. De hecho, una de las distinciones al poder hablar de interacción podría ser la que distingue entre la EAO clásica de los Tutores Inteligentes. Estos últimos intentan llevar a cabo una auténtica relación con el usuario, construyendo un modelo de él, llevando un cómputo de su historia previa de interacción, pudiendo acudir a módulos expertos para responder adecuadamente, etc. Aunque es cierto que en la actualidad los TI no se encuentran desarrollados y que existen dificultades tanto teóricas como técnicas para poder desarrollarlos completamente en el futuro, también lo es que son la auténtica alternativa en el campo educativo para hacer frente a la EAO más clásica, de corte skinneriano. La problemática de la interacción, y de sus grados, se sitúa en gran medida a este nivel.

Las diferencias entre la EAO y los TI son bien conocidas, si bien los puentes entre ambos tipos de software parecen ser hoy día una cuestión de tiempo. Como veremos, la progresiva integración del software va a permitir cada vez más que un programa de $\mathrm{EAO}$ realizado mediante lenguajes de autor pueda conectarse con un sistema de hipertexto para acceder a grandes masas de información contenidas por ejemplo en medios ópticos, e incluso utilizar el mismo sistema hipertextual como interfaz terminal de usuario para acceder a un sistema experto. Es en estos aspectos de integración donde más se tiene que avanzar y donde el sistema educativo debe poner grandes esperanzas.

\section{Los nuevos desarrollos}

\section{Los nuevos entornos de autor}

Junto a la cuestión genérica de la $\mathrm{EAO}$, lo cierto es que se han producido unos desarrollos técnicos muy importantes (sobre todo en software). Aunque no para las personas a él ajenas, el campo mismo de la microinformática educa- 
tiva ha cambiado mucho en los últimos años. Puede parecer una afirmación demasiado exagerada, sobre todo en terreno tan móvil, pero en modo alguno lo es: tiene nombres y fechas. Sólo centrándonos en el terreno específico de las herramientas para desarrollar software educativo, como hemos venido focalizando a lo largo de la exposición, y dejando de lado otros campos importantes (como, por ejemplo, el de la telemática), algunos productos muy recientes han cambiado el acceso técnico al campo de la producción de software educativo en microordenadores:

Antes de 1987: Primeras versiones de algunos paquetes. Pruebas.

1987: Guide, sistema de desarrollo de documentos hipertexto.

Hipercard, sistema de desarrollo en hipermedia, con lenguaje de programación incorporado (Hypertalk).

CourseBuilder, primer sistema de autor programable por iconos.

1988: Course of Action, sistema de autor programable por iconos.

IconAuthor, sistema de autor programable por iconos.

1989: LinkWay, sistema de desarrollo en hipermedia, con lenguaje de programación limitado.

Hyperpad, sistema de desarrollo, con lenguaje de programación limitado.

Plus. Supercard. Versiones más potentes de Hypercard.

1990: Director 2.0 (Interactivo), sistema de animación gráfica con capacidades interactivas y lenguaje de programación.

Esta lista no es precisamente exhaustiva ${ }^{4}$. Al menos una docena más de entornos de desarrollo han surgido en estos últimos años, aunque quizás no tan significativos ni tan interesantes para la educación.

¿Qué supone esto? Básicamente el acceso de los profesionales de la educación al nivel de autores de cursos en soporte informático. Esto es, se puede escribir muy fácilmente (mediante un lenguaje de programación basado en iconos) un buen número de programas que antes parecían labor exclusiva de los profesionales de la programación; o se puede crear un sistema de organización de la información textual (mediante Guide o Architext) o/y gráfica (mediante Hypercard) que permita al usuario la búsqueda de nuevas conexiones, que no sea lineal en su desarrollo, que le deje un cierto grado de libertad sobre lo que desea o no ver. Esto es, la mayoría de las operaciones básicas que un ordenador debería realizar en el terreno educativo parecen hoy accesibles para los profesionales de la educación y no sólo para los programadores o informáticos (más adelante lo comentaremos, si bien con algunas reservas importantes).

Pero, en segundo lugar, lo que también supone es la integración de las aplicaciones educativas a nivel de un sistema microinformático. Dicho de otra manera: estas aplicaciones pueden tener un efecto mucho mayor cuando se combinan que cuando se utilizan por separado, y existe la posibilidad de hacerlo de manera fácil. Cuando se abren pilas de Hypercard desde otros programas, o se utilizan gráficos animados como parte de un curso, o bien combinados en el proceso de búsqueda de información, etc., lo que se está haciendo es combinar tipos distintos de organización de la información con grados diferentes de interactividad, pero de manera que el usuario final sea ajeno a estas cuestiones técnicas y sólo compruebe lo que supone el poder acceder a varias fuentes de información integradas. La sensación final al utilizar un sistema integrado es muy distinta: el poder contemplar animación en determinados momentos, disponer de 
la facilidad intuitiva de un sistema de hipertexto cuando se requiere obtener información de una base de datos o de un CD-ROM, o pasar a un tutorial en el que se registran las respuestas del usuario, todo ello simultáneamente permite empezar a pensar que la potencia radical de la microinformática en la educación va precisamente por estos caminos.

\section{Multimedia interactivos}

Pero no sólo las nuevas aplicaciones van a ser determinantes en este replanteamiento. Quizá lo que va a tener un mayor impacto a medio plazo sobre la escuela va a ser la utilización del vídeo interactivo. A pesar de que no sea el momento para definiciones, entenderemos por vídeo interactivo (VI) el uso de material videográfico controlando por un ordenador (en el límite por un mando a distancia) mediante un programa que permite la congelación de la imagen, búsquedas rápidas, selecciones, etc., según los intereses/respuestas del usuario. El nivel o grado de interactividad puede ser mayor o menor, pero lo cierto es que con un ordenador controlado un vídeodisco un profesor puede organizar múltiples lecturas del mismo según distintos criterios, o los propios alumnos pueden ver el vídeodisco «desde» un curso creado con un lenguaje de autor, accediendo así a distintas partes del mismo en función de sus respuestas individuales, etc.

En el mercado del software para control de vídeodisco han aparecido un buen número de paquetes para entornos DOS. Ya a mediados de 1987 una revista especializada comparaba 7 paquetes. Lo más característico es que se trata de lenguajes de autor sencillos, en algunos casos incluso pobres desde el punto de vista de sus capacidades para facilitar la labor de autoría, que han incorporado un conjunto de instrucciones para el control de vídeodisco. Sin embargo, debemos tener muy claro que por el momento el VI es una herramienta destinada casi exclusivamente al mundo empresarial. Ello es así por varios motivos:

a) el alto precio de los LA que controlan vídeodiscos en el ámbito de MsDOS (aunque hay LA que son asequibles, lo cierto es que la mayoría son muy caros).

b) la necesidad de utilizar vídeodisco en lugar de vídeocinta convencional. Los diferentes tiempos de acceso condicionan la interactividad en gran medida ( 2 segundos para el vídeodisco, entre 10 segundos y dos minutos para la cinta). Sin embargo, sería posible al menos teóricamente utilizar un diseño que optimizase las secuencias de búsqueda y que permitiese reducir la espera considerablemente. Otra cuestión asociada es la facilidad de control de un vídeodisco y las dificultades para hacerlo con una vídeocinta. Se trata de un problema que se resolverá con modelos más perfeccionados de VHS y $8 \mathrm{~mm}$ (aunque ya existen algunos que son controlables, al poder determinar exactamente el marco de referencia).

c) el alto precio exigible en la producción de un vídeodisco. Por el momento, el trabajo con vídeodisco requiere la realización de un master y la obtención posterior de copias. Este proceso es algo más barato en el caso de otros medios ópticos (como CD-ROM), pero que todavía no han acabado de resolver las necesidades técnicas de la información que deben contener (imágenes estáticas, vídeo, sonido digitalizado, soporte de color, etc.). Es de suponer que la comercialización de vídeodiscos profesionales con capacidad de grabación va a per- 
mitir la producción de un buen número de prototipos experimentales a precios mucho más aceptables.

En resumen, que el VI exige la realización de vídeodiscos y que éstos sólo pueden ser financiados por empresas (para la formación de su propio personal) o por organismos públicos (Gobiernos, Ayuntamientos, Fundaciones, etc.), a la vez que es necesario contar con vídeodiscos lectores. Sin embargo, sea bajo la forma que finalmente predomine, lo cierto es que el control de medios ópticos por ordenador, así como las posibilidades de interacción que permiten, va a in. fluir cada vez (aunque sea de manera lenta por la envergadura económica) sobre el tipo de recursos informáticos que se utilizan en educación.

Dejamos este tema sin apenas desarrollar. La cuestión del control de medios ópticos (no sólo el vídeodisco) y la interacción resultante plantea la cuestión más general de los multimedia. Los productos multimedia son el resultado de aplicar las nuevas tecnologías (ordenadores, medios ópticos,...) sobre una materia prima más convencional (texto, material sonoro, audiovisuales), creando algo nuevo, profundamente interactivo, que combina ambos tipos de tecnologías en algo diferente (por ej., novelas interactivas, tutoriales por vídeodisco, formación por vídeodisco controlado por un Lenguaje de Autor, simulaciones, etc.). El siguiente esquema, ligeramente retocado de Ambron (1988) para ser algo menos lineal, ejemplica este proceso de producción de nuevos productos (de momento, como siempre, más ligados al campo industrial que al educativo):

\begin{tabular}{|c|c|}
\hline $\begin{array}{c}\text { MEDIA } \\
\text { (Materia Prima) }\end{array}$ & $\begin{array}{c}\text { TECNOLOGIAS } \\
\text { (Medios de Producción) }\end{array}$ \\
\hline TEXTO & $\begin{array}{l}\text { Ordenador } \\
\text { AUDIO } \\
\text { VÍDEO } \\
\text { Vídeodisco Opticos: } \\
\text { CD-ROM }\end{array}$ \\
\hline
\end{tabular}

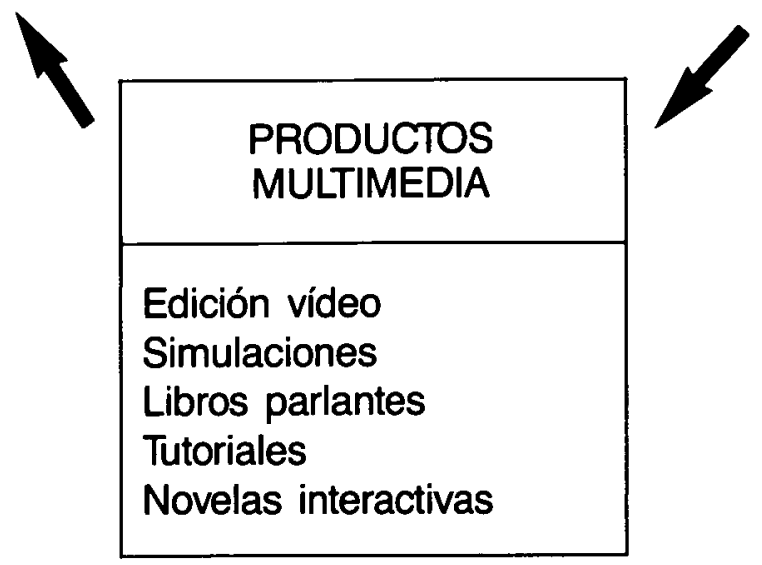




\section{Simulación}

Lo que todos los enseñantes están de acuerdo en considerar como uno de los aspectos más claros de la EAO son las simulaciones. No tanto las simulaciones muy abstractas de sistemas dinámicos (que también podríamos considerar), cuanto las simulaciones de sistemas reales: por ejemplo, los juegos clásicos de simulación de pequeñas empresas en los que el usuario debe manejar sus recursos económicos, hacer inversiones en stock, en propaganda de lo que vende, debe a la vez tener siempre un mínimo de líquido para hacer frente a los pagos urgentes, etc. Bajo el formato de juegos de ordenador se han introducido muchas simulaciones (de hecho, como ya señalamos, la forma básica de la simulación es el juego), aunque también otras han adoptado un enfoque claramente didáctico; por ejemplo, el programa Electric, para PC's, desarrollado por Idealogic es una representación realista de toda la panoplia de instrumental eléctrico clásico de los juegos didácticos: bombillas, generadores, voltímetros, etc., con los que el usuario puede montar sus propios circuitos y comprobar si funcionan bien. Lo que se ha hecho es crear un entorno de simulación mediante la matematización de las leyes básicas de la electricidad (en otros casos es de la química, del magnetismo, etc.). De alguna manera simplificada el ordenador se convierte en un laboratorio electrónico, desplazando la manipulación real de los cables y bombillas por los correspondientes iconos que son recolocados en el interior de redes o circuitos mediante el ratón. Es obvio que la fuerza de este tipo de EAO es la modelización de las leyes a la que nos hemos referido. No sería lo mismo intentar modelizar otros aspectos menos matematizables y que también son transmitidos por la escuela, aunque también hay que considerar que se pueden construir más simulaciones de las que habitualmente se piensa: aunque no son muchos los ejemplos, podríamos considerar el caso de algún programa que intenta situarnos en una época histórica determinada (p.e., Would Be Gentleman) y ver cómo nos desenvolvemos a lo largo de los años; las costumbre de la época deben ser aprendidas, las inversiones deben realizarse, etc.

Pero, sin embargo, la simulación aparece donde menos se espera: pensemos, por ejemplo, en el diseño gráfico, o en el programa de dibujo que revolucionaron los micros y el tratamiento de la imagen hace apenas 5 años. Típicamente es el caso de MacPaint y sus múltiples derivados, o de las versiones actuales en color, p.e., y entre otros, PixelPaint. Lo que sorprende y maravilla de estos programas es la metáfora tan ajustada que han conseguido de una actividad tan aparentemente incontrolable como es el dibujo; metáforas del lápiz, de la goma, del tiralíneas, del rotulador o brocha, del relleno total de una forma, de las formas predefinidas, de la multitud de efectos especiales sólo conseguibles mediante el ordenador, metáfora incluso del compás. No es de extrañar que si la pantalla es una hoja en blanco y el software simula todas las herramientas de un estudio de un diseñador/pintor los resultados hayan sido tan espectaculares: sencillez de uso, potencia de realización, ausencia de castigo por el error (existe una opción por la que se vuelve a la situación justo anterior a haberse equivocado). Lo más sorprendente sin embargo es ver a niños pequeños enfrentados con estos programas: los prefieren a la mayoría de juegos y software especialmente creados.

¿Acaso no son los programas más populares, los procesadores de texto, perfectas simulaciones/mejoras de la máquina de escribir? Sólo en cierto sentido, desde luego, pero no más alejado que cualquier otra aplicación de uso general: la creación de una simulación electrónica de algo que se hace en la vida cotidiana. 


\section{Otros desarrollos}

En estos años se están produciendo también otros desarrollos no menos importantes que los mencionados: asentación progresiva de los principios de la inteligencia artificial en forma de técnicas utilizadas amplia y habitualmente, incorporación de la comunicación telemática en diversas esferas de la vida cotidiana y de la educación, avances importantes en la aplicación al campo de la educación especial, etc. Todos ellos, y muchos otros incluyendo los cambios en el hardware, van a jugar un papel sinérgico en el despegue definitivo de los usos $\mathrm{y}$ adecuaciones de la informática en la educación tanto formal como no formal.

El foco de este artículo ha sido más mostrar cómo están cambiado algunos elementos estratégicos antes que dar una visión de conjunto de un campo demasiado disperso. Si el enfoque es correcto, los próximos años supondrán un asentamiento progresivo en la utilización de los ordenadores como herramienta educativa propia -además de seguir usándolos como máquinas de propósito general-, y las polémicas que lo han precedido serán olvidadas rápidamente. En buena parte será debido a los importantes avances en desarrollo de hardware y especialmente de software dedicado a la producción de materiales didácticos, lo que posibilitará que el eje de las discusiones comparta mucho más que en la actualidad consideraciones técnicas sobre aspectos informáticos con consideraciones pedagógicas (las relativas al diseño instructivo, a su inserción curricular, etc.).

\section{Anexo}

\section{HIPERTEXTO E HIPERMEDIA}

El concepto de hipertexto, y por extensión de hipermedia, está siendo cada día más invocado en ámbitos muy distintos (VV.AA. 1988, Rodríguez de las Heras 1988). Sin embargo, la idea no es precisamente nueva. Ya en 1945, Vannevar Bush (1945) planteó por vez primera la existencia de un sistema de organización de la información que podríamos denominar «hipertexto». Posteriormente, sólo a finales de los años 60 , a través de las ideas de Ted Nalson, el asunto se volvió a plantear con la intención de realizarlo (de hecho, de hecho, fue Nelson quien inventó la palabra hipertexto, y quien realizó uno de los primeros sistemas hipertextuales, Xanadú). Desde ese momento, y muy lentamente, se han venido implementando algunos sistemas de hipertexto; por ejemplo, NoteCard. Una característica común a todos ellos es que han utilizado estaciones de trabajo, y que han sido desarrollados por grandes industrias ligadas a la propia informática (Xerox) o por universidades para uso interno (por ej., la Brown University y su proyecto Intermedia); esto ha hecho que sólo muy recientemente se haya empezado a hablar de estos sistemas en el contexto de microodenadores y, por consiguiente, en el educativo.

Básicamente un sistema hipertextual es aquel en el que la información contenida está relacionada. Pero no como en una base de datos, mediante campos y relaciones construidas, sino en todos y cada uno de los aspectos que el creador del sistema decida que deban relacionarse. La técnica consiste en crear unas zonas en pantalla (¡de 1 pixel como mínimo!) que podríamos denominar «sensi- 
bles», y que son auténticos nudos o nodos que relacionan información situada a distintos niveles. Un «click» sobre una de estas zonas es suficiente para que aparezca en pantalla la información original. Como ya parece todo esto suena a teoría de grafos, y quizás sea esa la mejor manera de pensar en un sistema de hipertexto: un gigantesco grafo n-dimensional que podemos recorrer en muy distintas maneras. Otra manera un poco más exagerada sería comparar un hipertexto a un diccionario: cualquier palabra nos lleva a cualquier otra, a través de otras muchas palabras.

La idea de hipermedia suele conllevar todavía algo más: la apertura del sistema a información no sólo textual sino de cualquier otro tipo. Más aún: la integración real de varios media en un único entramado hipertextual. Una aplicación para micros, Hypercard (Goodman, 1987, 1988), ha sido el punto de lanzamiento de estos nuevos conceptos. No sólo por su interfaz absolutamente gráfico (además de un potente editor gráfico incorporado), sino por la complejidad de los lazos interinformativos que pueden realizarse: al añadir un lenguaje de programación, Hipertalk, a las zonas sensibles de conexión, aquí llamadas abotones», el resultado es que la relación puede ser definida con toda la complejidad e individualidad que se quiera. Más aún, Hypercard se está mostrando como el interfaz más adecuado para acceder a enormes masas de información así como para el control de: vídeodiscos, CD-ROM, digitalizadores gráficos, digitalizadores de sonido, interfaz con sistemas expertos.

Las siguientes imágenes son copias de pantallas de Hypercard y pueden dar una idea más exacta del tipo de interfaz gráfico e intuitivo (con botones en forma de flechas, campos de información, gráficos, etc.):

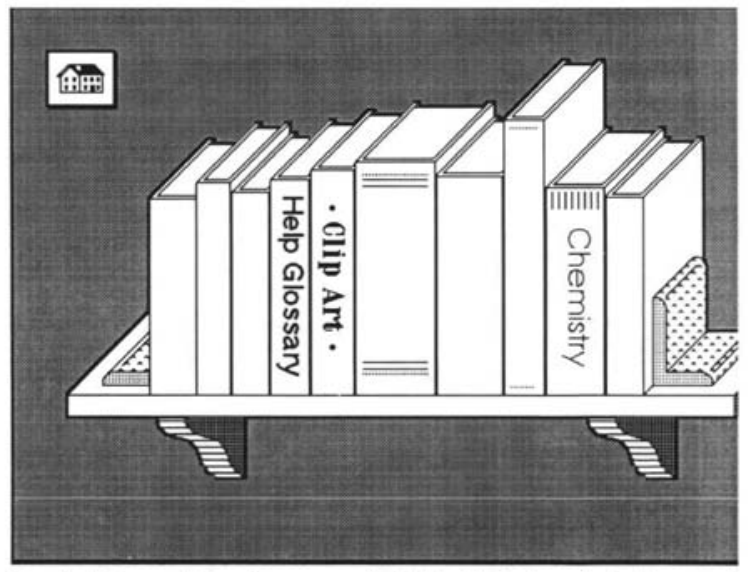

Pantalla de Hypercard: Una tarjeta que es un distribuidor de pilas (o estackso) o de otras tarjetas bajo la metáfora de un estante con libros. Aquellos que contienen un título son de hecho botones que al ser señalados y pulsados envían de manera inmediata a la tarjeta o/y pila correspondiente. El botón de arriba, una casa pequeña, remite siempre a un punto de referen. cia intocado (ahomes). 
Uno de los aspectos más interesantes es que el paso de una actividad a otra, la unión entre las dos más alejadas, no supera nunca uno o dos segundos. Y aquí una disponibilidad técnica se convierte en consecuencia educativa: la inmediatez es interacción.

Esta caja de herramientas tan versátiles y tan potentes hace que los hipertextos y los hipermedia sea la forma más sencilla de «navegar» a través de grandes masas de conocimiento (según una metáfora, Knowledge Navigator, que ha sido célebre a lo largo de 1988 en la industria de microordenadores). No cabe duda que gran parte de la aplicación de la informática en los ámbitos educativos y domésticos del próximo futuro pasa precisamente por el control de medios ópticos, así como por permitir la búsqueda personal en bases de datos cada vez mayores. El hipertexto es interconexión y en ese sentido sólo cabe apuntarse a este avance.

A pesar de ello, también es cierto que se trata de un avance tecnológico todavía poco maduro; no tanto a nivel de microordenadores, o como filosofía para adaptar el interfaz de usuario final a grandes masas de información, sino en grandes sistemas de hipertexto (o, desde luego, en lo referente a sus propias posibilidades educativas, cfr. Carr, 1988): en ellos es necesario un mantenimiento de la información, del propio sistema, de las conexiones nuevas que se crean, etc., que puede plantear problemas importante. El propio interfaz de usuario/gráfico es un tema complejo (Canals y Llàcer, 1990), así como la existencia de varios sistemas muy diferentes para generar documentos hipertextuales.

\section{Notas}

${ }^{1}$ Por desgracia, no se dispone de estadísticas sobre la procedencia social de los compradores de micros.

2 Este breve listado, que por su propia generalidad debe necesariamente ser inexacto, da creemos una buena idea de lo que es un vector constante de las críticas recientes a los modos de producción e inserción curricular del software educativo clásico: la complejidad a veces insuperable en el proceso de producción, su carácter cerrado y poco flexible para el profesorado, y, en fin, su poca «transportabilidad» a unidades y áreas curriculares otras para aquellas que fue creado. Estas críticas que nos interesa enfatizar aquí se añaden a las ya bien conocidas sobre la calidad/adecuación a sus objetios de la denominada enseñanza asistida por ordenador y que no vamoa a abordar aquí en detalle. La mayoría de ellas señalan el carácter abstracto y descontextualizado en relación a la tarea del software educativo, de la inexistencia o mala modelización del alumno que suelen llevar, de su carácter poco o nada inteligente, del débil diseño instructivo con el que han sido realizadas, etc. Entre otros, cfr. Johanssen (ed, 1988), Lawler y Yazdany (ed, 1988).

${ }^{3}$ La cuestión del interfaz de comunicación persona-máquina está convirtiéndose, día a día, en fundamental. Y no sólo por los tipos y formas de entrada de datos, sino por todo el trabajo de ingeniería psicológica subyacente al diseño de las acciones que el usuario puede realizar «con» la pantalla. Este ha sido el gran mérito de los denominados VGI, interfaces gráficos, iniciados por Xerox, desarrollados y teorizados principalmente por Apple con los ordenadores Lisa y Macintosh, y punto de encuentro de todas las máquinas en la actualidad, desde los basados en Ms-DOS a través de entornos GEM y Windows, hasta los que trabajando en UNIX adoptan unas formas gráficas de comunicación (Next, X-Windows,). Las referencias son ya muy amplias: Apple Computer (1988), Barker (1989), Sutcliffe (1988), etc.

${ }_{4}^{4}$ Para un análisis más detallado de los entornos de autor, Rodríguez Illera (1990).

\section{Referencias}

AMBron, S. (1988): «Introduction», in Ambron y Hooper (comps, 1988), 1-11.

Ambron, S., y Hooper. K. (comps. 1988): Interactive Multimedia, Redmon, WA, Microsoft Press, 1988. 
Apple Computer (1987): Human Interface Guidelines. Addison-Wesley, 1987.

BARKER, P. (1988): Basic Principles of Human-Computer Interface Design. London, Hutchinson, 1988.

Brand, S. (1987): The Media Lab. Inventing the Future at M.IT., New York, Penguin, 1988.

Busn, V. (1945): «As we may think», in S. Lambert y S. Ropiequet (comps., 1986): CD-Rom: El nuevo papiro. Madrid, Anaya, 1988.

Canals, I. , y Llàcer, E. (1990): «Repórter Olímpic. Por una metodología de diseño de interfaces "navegacionales" para los sistemas hipertexto basada en el análisis de facetas", in Insys' 90. Jornades de noves tecnologies interactives, Barcelona, Fundació Caixa de Pensions, 1990, 137-156.

CARR, C. (1988): «Hypertext: A New Training Tool?», in Educational Technology, XXVIII, $n$. 8, 7-11, 1988.

Goodman, D. (1987): The Complete Hypercard Handbook. New York, Bantam, 1988, 2.a ed. aumentada.

Goodman, D. (1988): Hypercard Developpers Guide. New York, Bantam, 1988.

Gros SAlvar, B. (1987): Aprender mediante el ordenador. Barcelona, P.P.U., 1987.

Gros Salvat, B. , y Rodríguez Illere, J.L. (1985): Evaluación de software educativo. Cuadernos de Estudio, 3, 1986, Dpto. de Pedagogía Sistemática, Universidad de Barcelona.

Johanssen, D. (ed, 1988): Instructional Desing for Microcomputers Courseware. Lawrence Erlabaum, 1988.

KING, D. (1989): «La aplicación del software en la Educación Especial», Comunicación, Lenguaje y Educación, 5, 1990, 31-46.

Lawler, R.W. , y YazDANI, M. (comps, 1987): Artificial Intelligence and Education, vol. 1., Norwood, Ablex, 1987.

Pfeffer, A. , y Gawún, J. (comps., 1985): Informática y escuela. Madrid, Fundesco, 1985.

Rodriguez De Las Heras, A. (1988): «La enseñanza de la Historia asistida por ordenador. Las posibilidades del hipertexto-set», documento fotocopiao, a publicar por la editorial Laia, Barcelona.

Rodríguez Illera, J.L. (1986): «L'Enseyament de la Informàtica. Programadors o Usuaris?», in M.A.A guareles et al. (comps, 1988): L'educació davant la informàtica, Barcelona, P.P.U., 1988.

Rodriguez Illera, J.L. (1990): «Instructional Design and Authoring Environmentsm, Delta and Beyond Conference, The Hague, Octubre 1990.

Sutcurfe, A. (1988): Human-Computer Interface Design. London, MacMillan, 1988.

VV.AA. (1988): Hypertext. Informe de la revista Byte, Octubre, 1988, pp. 234-269.

La informática educativa:

Presente y Futuro.

José Luis Rodríguez.

CL\&E, 1992, 13, pp. 51-72

Resumen: Se analizan los problemas de desarrollo de la informática educativa, así como los cambios que se han producido en las herramientas que se utilizan, tanto las máquinas como los programas. El artículo es una defensa de los denominados «interfaces gráficos de usuarion y de los nuevos campos aplicados que se están abriendo (multimedia, inteligencia artificial, etc.), y de sus potencialidades para la educación.

Datos sobre el autor: José Luis Rodríguez Illera trabaja en el Instituto de Ciencias de la Educación, División de Ciencias de la Educación, de la Universidad de Barcelona.

Dirección: Universidad de Barcelona. Carrer dels Angels, 18. 08001 Barcelona.

(C) De todos los artículos. Deberá solicitarse por escrito autorización de CL\&E y de los autores para el uso en forma de facsímil, fotocopia o cualquier otro medio de reproducción impresa. CL\&E se reserva el derecho de interponer las acciones legales necesarias en aquellos casos en que se contravenga la ley de derechos de autor. 